\title{
Potential Targeting of Siglecs, Mast Cell Inhibitory Receptors, in Interstitial Cystitis
}

\author{
Chang-Shin Park ${ }^{1,2}$, Bruce S. Bochner ${ }^{3}$ \\ ${ }^{1}$ Department of Pharmacology, Inha University School of Medicine, Incheon; \\ ${ }^{2}$ Medicinal Toxicology Research Center, Inha Institute of Research for Medical Sciences, and Center for Advanced Medical Education, Inha University College \\ of Medicine by BK-21 Project, Inha University School of Medicine, Incheon, Korea \\ ${ }^{3}$ Division of Allergy and Clinical Immunology, Department of Medicine, Johns Hopkins University School of Medicine, Baltimore, MD, USA
}

\begin{abstract}
Mast cell increases and activation are detected in the chronic inflammatory bladder disease interstitial cystitis (IC), and their proinflammatory mediators are felt to contribute to regional pelvic pain and inflammatory pathophysiology. The immunoreceptor tyrosine-based inhibition motif-containing sialic acid-binding immunoglobulin-like lectins (Siglecs) expressed in mast cells could be evaluated as in vivo signaling regulators capable of inhibiting IC-related mast cell activation.
\end{abstract}

Keywords: Urinary bladder; Interstitial cystitis; Mast cells; Sialic acid binding Ig-like lectin (Siglec)

Interstitial cystitis (IC) is a chronic bladder inflammatory disease that is characterized by symptoms such as urinary urgency, frequency, nocturia, and suprapubic and pelvic pain and that mainly affect women. Although the exact etiology and pathogenesis of IC is unknown, one etiologic theory that has been dominantly reported in relation to the bladder immune is that of a localized allergic reaction $[1,2]$. As reviewed by Theoharides et al. [1] and Rudick et al. [2], both clinical and experimental evidence show that IC is closely associated with an accumulation of mast cells and their activation, especially in the detrusor, lamina propria, and submucosa. This theory was first introduced after the beneficial therapeutic use of antihistamines in IC patients [3]. Recently, detrusor mast cell migration and activation and urinary histamine metabolites have been detected in IC patients and in experimentally induced animal models [4-6]. Concerning these points, both the bladder pathophysiology and pelvic pain are dependent on mast cells. However, whereas pharmacological modulators of histamine receptors can be evaluated as therapeutic agents to improve chronic pelvic pain, they do not act on the pathophysiology, which is mediated by tumor necrosis factor (TNF)- $\alpha$. These results indicate different causative factors for bladder pain and the pathophysiological inflammation [2].

Mast cells are well known to be involved in both acute and chronic forms of allergic, type I hypersensitivity reactions. Mast cells are highly granulated cells that populate tissues including muscles and mucosal surfaces. Mast cells matured in these tissues are partially or completely degranulated by a wide range of immunologic and nonimmunologic stimuli. After activation, a characteristic pattern of mast cell mediators is released during degranulation. These include preformed granule-associated inflammatory mediators (e.g., histamine, heparin, neutral proteases, proteoglycans, glycoprotein-like YKL-40, chemotactic substances, and cytokines such as TNF- $\alpha$ ), newly synthesized mediators (e.g., prostaglandin D2 [PGD2], leukotriene B4 and C4, interleukin-6 [IL-6], platelet-activating factor, and nitric oxide), and potent vasodilatory mediators (e.g., vasoactive intestinal peptide) $[1,7,8]$. These mast cell-released mediators are implicated in causing IC bladder pathophysiology (e.g., apoptotic lesions in the urothelium and urothelial or mucosal barrier dys-
Corresponding author: Chang-Shin Park

Department of Pharmacology, Medicinal Toxicology Research Center and Center for Advanced Medical Education, Inha University School of Medicine, 7-206 Sinheung-dong 3-ga, Jung-gu, Incheon 400-712, Korea

Tel: +82-32-890-0962 / Fax: +82-32-887-7488 / E-mail: parkshin@inha.ac.kr

Submitted: June 10, 2011 / Accepted after revision: June 20, 2011
This is an Open Access article distributed under the terms of the Creative Commons Attribution Non-Commercial License (http://creativecommons.org/licenses/by-nc/3.0/) which permits unrestricted non-commercial use, distribution, and reproduction in any medium, provided the original work is properly cited. 
function) and bladder-associated pelvic pain. Furthermore, the serum and urine levels of a new biomarker YKL-40, which is expressed in bladder mast cells, can be evaluated as an inflammatory mediator of bladder fibrogenesis in IC patients [8]. Also, IL-6 released from mature mast cells is elevated in the urine of IC patients and is highly increased in such patients with severe inflammation [9]. Thus, direct control via mast cell death or physiological blockade of bladder mast cell function is a reasonable strategy for reducing the severity of IC.

Mast cell survival and activation, which are mediated mainly by phosphorylation signals, can be negatively regulated by engaging the inhibitory receptors (IRs) containing immunoreceptor tyrosine-based inhibitory motifs (ITIMs). Upon activation, the tyrosine residues of the ITIM sequences of the IRs are phosphorylated by the Src family of protein kinases [10]. The tyrosine phosphorylation typically induces recruitment of phosphatases, such as SHP-1 and SHP-2, and SH2-containing inositol phosphatases to the plasma membrane, which are then activated. Mast cell degranulation, for example as induced via FceRI, is inhibited by this phosphatase-induced dephosphorylation mechanism [11].

A classic form of several IRs on human mast cells is Fc $\gamma$ RIIB, which can down-regulate mast cell activation when co-ligated with high-affinity IgE receptor (FceRI) [7]. In addition to the classic Fc $\gamma$ RIIB form, mast cells also express one or more ITIM sequence-containing IRs, such as sialic acid-binding immunoglobulin-like lectins (Siglecs), on the cell surface to regulate their inflammatory or allergic activation. In this short review, we introduce the potential application of Siglec antibodies to the mast cells to activate Siglec IRs in the inflammatory urinary bladder disease IC.

Siglecs are members of an immunoglobulin superfamily. They are type 1 transmembrane proteins containing a unique $\mathrm{N}$-terminal 'V-set' Ig domain that can bind sialic acid and variable numbers of extracellular 'C2-set' Ig domains. Most Siglecs (CD22 [Siglec-2]- and CD33 [Siglec-3]-related forms) also have a conserved cytosolic domain containing single or repeated ITIMs or ITIM-like motif sequences that are actively phosphorylated and that display inhibitory signaling functions. In particular, the genes for the CD33-related Siglecs (Siglec-3, -5, -6, -7, $-8,-9$, and -10 ) are located on chromosome 19 and have one or two ITIM sequences [12-14]. These Siglecs have been implicated mainly in the functional regulation of leukocytes via triggering signals via their ITIMs. Indeed, an important role of the CD33-related Siglecs is to regulate cell expansion by inhibiting or inducing apoptosis in leukocytes, but there are no reports of such activity in mast cells.

In human mast cells, CD22 (Siglec-2), CD33 (Siglec-3), and Siglecs-5 though $-10[15,16]$ are expressed at differential levels. In particular, the expression of the eosinophil-selective Siglec-8 is also found weakly on basophils but prominently on mast cells. In functional experiments, activation of Siglec- 8 by a specific monoclonal antibody $(\mathrm{mAb})$ induced eosinophil apoptosis via caspase- and reactive oxygen species (ROS)-dependent processes. In contrast, these antibodies failed to induce mast cell apoptosis but did inhibit by at least 50\% histamine and PGD2 release triggered via FceRI. Additionally, in studies with human mast cells and Siglec- 8 transfected RBL cells, activation (crosslinking) of Siglec-8 with $\mathrm{mAb}$ inhibited IgE receptor-mediated beta hexosaminidase release and calcium influx, the latter of which is required for mast cells degranulation $[17,18]$. Indeed, normal human mast cells like LAD2 cells dominantly express Siglec- 6 and -7 and moderately express Siglec- 3 and -5 , although expression is variable according to the culture stages of the mast cells. In our in vitro preliminary study in LAD2 cells, crosslinking of each of these Siglecs before FceRI-stimulation, or simultaneously co-crosslinking a given Siglec mAb and IgE, partially inhibited histamine secretion [unpublished data]. As suggested also by Bochner [19], activation of Siglecs other than Siglec-8 would be expected to inhibit ITAM (immunoreceptor tyrosinebased activating motif)-dependent secretory responses in mast cells. Therefore, activating mast cell Siglecs with specific mAbs, or glycan liagnds specific for a particular Siglec) could be used to down-regulate mast cell function.

Increased numbers and activation of urinary bladder mast cells are major contributors to the pathophysiology of both ulcer and non-ulcer IC and in toxin- or chemical-induced animal models of IC. Although there are no reports targeting Siglecs or other mast cell IRs as a treatment for IC, the identification of these IRs on bladder infiltrated-mast cells would further our understanding of the mast cell phenotype in this disease, and may provide enthusiasm for the utilization of Siglec-specific $\mathrm{mAbs}$ or their glycan ligands to deliver inhibitory signals in vivo to mast cells as a potential treatment to reduce IC-induced bladder mast cell expansion and activation.

\section{REFERENCES}

1. Theoharides TC, Kempuraj D, Sant GR. Mast cell involvement in interstitial cystitis: a review of human and experimental evidence. 
Urology 2001;57(6 Suppl 1):47-55.

2. Rudick CN, Bryce PJ, Guichelaar LA, Berry RE, Klumpp DJ. Mast cell-derived histamine mediates cystitis pain. PLoS One 2008;3: e2096.

3. Simmons JL, Bunce PL. On the use of an antihistamine in the treatment of interstitial cystitis. Am Surg 1958;24:664-7.

4. Sakthivel SK, Singh UP, Singh S, Taub DD, Novakovic KR, Lillard JW Jr. CXCL10 blockade protects mice from cyclophosphamideinduced cystitis. J Immune Based Ther Vaccines 2008;6:6.

5. Tomaszewski JE, Landis JR, Russack V, Williams TM, Wang LP, Hardy C, et al. Biopsy features are associated with primary symptoms in interstitial cystitis: results from the interstitial cystitis database study. Urology 2001;57(6 Suppl 1):67-81.

6. el-Mansoury M, Boucher W, Sant GR, Theoharides TC. Increased urine histamine and methylhistamine in interstitial cystitis. J Urol 1994;152(2 Pt 1):350-3.

7. Li L, Yao Z. Mast cell and immune inhibitory receptors. Cell Mol Immunol 2004;1:408-15.

8. Richter B, Roslind A, Hesse U, Nordling J, Johansen JS, Horn T, et al. YKL-40 and mast cells are associated with detrusor fibrosis in patients diagnosed with bladder pain syndrome/interstitial cystitis according to the 2008 criteria of the European Society for the Study of Interstitial Cystitis. Histopathology 2010;57:371-83.

9. Erickson DR, Belchis DA, Dabbs DJ. Inflammatory cell types and clinical features of interstitial cystitis. J Urol 1997;158(3 Pt 1):790-3.

10. Bolland S, Ravetch JV. Inhibitory pathways triggered by ITIM-containing receptors. Adv Immunol 1999;72:149-77.
11. Tamir I, Dal Porto JM, Cambier JC. Cytoplasmic protein tyrosine phosphatases SHP-1 and SHP-2: regulators of B cell signal transduction. Curr Opin Immunol 2000;12:307-15.

12. Crocker PR, Paulson JC, Varki A. Siglecs and their roles in the immune system. Nat Rev Immunol 2007;7:255-66.

13. von Gunten S, Bochner BS. Basic and clinical immunology of Siglecs. Ann N Y Acad Sci 2008;1143:61-82.

14. O'Reilly MK, Paulson JC. Siglecs as targets for therapy in immunecell-mediated disease. Trends Pharmacol Sci 2009;30:240-8.

15. Ghannadan M, Hauswirth AW, Schernthaner GH, Müller MR, Klepetko W, Schatzl G, et al. Detection of novel CD antigens on the surface of human mast cells and basophils. Int Arch Allergy Immunol 2002;127:299-307.

16. Yokoi H, Myers A, Matsumoto K, Crocker PR, Saito H, Bochner BS. Alteration and acquisition of Siglecs during in vitro maturation of CD34+ progenitors into human mast cells. Allergy 2006;61:769-76.

17. Nutku E, Aizawa H, Hudson SA, Bochner BS. Ligation of Siglec-8: a selective mechanism for induction of human eosinophil apoptosis. Blood 2003;101:5014-20.

18. Yokoi H, Choi OH, Hubbard W, Lee HS, Canning BJ, Lee HH, et al. Inhibition of FcepsilonRI-dependent mediator release and calcium flux from human mast cells by sialic acid-binding immunoglobulin-like lectin 8 engagement. J Allergy Clin Immunol 2008; 121:499-505.e1.

19. Bochner BS. Siglec-8 on human eosinophils and mast cells, and Siglec-F on murine eosinophils, are functionally related inhibitory receptors. Clin Exp Allergy 2009;39:317-24. 